\title{
PRIORITY SCHEDULING FOR MULTIPATH VIDEO TRANSMISSION IN WMSNS
}

\author{
Elham Karimi ${ }^{1}$ and Behzad Akbari ${ }^{2}$ \\ ${ }^{1}$ Department of Electrical and Computer Engineering, Qazvin Islamic Azad University, \\ Qazvin, Iran \\ ${ }^{2}$ Department of Electrical and Computer Engineering, Tarbiat Modares University, \\ Tehran, Iran
}

\begin{abstract}
In video data transmission over Wireless Multimedia Sensor Networks (WMSNs), in which network congestion and link failures are main characteristics, providing Quality-of-Service $(Q o S)$ is challenging. In such situation, using multipath routing protocols along with efficient packet scheduling can improve the performance of video delivery over sensor networks. In this paper we propose a new packet scheduling mechanism for multipath video transmission over WMSN. Since, different video frame types in a compressed video stream have different impact on the perceived video at the receiver; our proposed scheme for video packet scheduling is content aware that schedules transmission of different video packets over different paths. For this purpose, condition of each routing path is periodically evaluated and the high priority video packets are transmitted through high quality paths. Simulation results show that our proposed scheduling improves the quality of the perceived video at the receiver, considerably.
\end{abstract}

\section{KEYWORDS}

Multimedia Sensor Networks, Video Transmission, Packet Scheduling, Multipath Routing

\section{INTRODUCTION}

Wireless sensor networks (WSNs) are self-organizing networks having very large number of sensor nodes. These nodes are usually scattered over an environment to collect information from the perimeter. They have properties such as low cost, small size and weight, and due to their limited resources, i.e. energy, memory, and processing power, they must be placed closely to each other [1,2]. WSNs are subject to advancement because of their simplicity and applicability. Furthermore, new applications have been developed for them. Over the last few years, Wireless Multimedia Sensor Networks (WMSNs) have been employed for multimedia communication within a sensor network. WMSNs are able to retrieve multimedia content such as video and audio streams, still images, and scalar sensor data from the environment $[1,2]$.

There are many challenges to be addressed to support video communication over sensor networks. Decreasing delay in real-time video streaming is a crucial problem. In addition, large amount of bandwidth is needed for video transmission [3, 4]. Hence, routing protocol plays an important role for optimizing network throughput, efficient bandwidth usage and decreasing endto-end delay. Several routing protocols are proposed for these goals. Most of data transmission protocols only use an optimal single path. To detect such path, some criteria should be considered like data transmission direction, the distance from the destination node, and the remaining energy DOI : 10.5121/ijcnc.2013.5610 
level in each node [5]. In this sort of protocols, when a main path is identified between the source and destination nodes, the data would be transmitted through the distinguished path.

Using single path for transmission of a bandwidth demanding video traffic in a sensor network with limited energy nodes causes the energy of the nodes in the path to be consumed and finished sooner than other nodes of the network that exterminates the accessibility of the nodes in the neighbourhood of the path after a while. In addition, due to the error prone nature of wireless channels, using single path may decrease the reliability of data transmission in the network [5].

Unlike single path, multipath transmission increases bandwidth efficiency and reliability, and decreases end-to-end delay of video delivery over WMSNs. A class of routing protocols entitled as "multiple path routing" presents a group of routing algorithms which allow every source node to identify some different paths toward the destination node for data transmission [3,5]. Using this technique, respecting the quality of each path, the network traffic can be shared on several paths. Hence the system's resources would be used in an optimal manner and the required quality of services will be provided for transmission of video contents too. The multipath routing is used for various purposes in different networks, such as: improving the reliability and resiliency, decreasing the network traffic and decreasing the delay [6, 7, and 8]. But the existing restrictions of WMSN, causes the need to some new essentials in order to use these techniques for video transmission.

The problem of video streaming with resource constraints in WMSN proves challenging as network traffic increases, thus a packet scheduling mechanism can play important role in optimizing the quality of video streaming applications over WMSNs. In this paper we propose a packet scheduling mechanism for multipath video transmission over a sensor network in which the video packets are scheduled based on multiple paths properties and also based on video packets content. Since different video frames in a compressed video stream have different impact on perceived video quality at the receiver, our proposed mechanism takes into account the type of video packets in scheduling them for transmission over multiple paths and also queue scheduling at the source node [3, 4 and 5].

The remaining of this paper has been organized as following: We review the related works in Section 2. Section 3 describes the proposed priority scheduling model. The performance evaluation of the proposed mechanism through simulation results are provided in section 4. Finally, we conclude the paper in Section 5.

\section{RELATED WORKS}

Several packet scheduling solutions have been proposed for video transmission over MWSNs. The work in [9] has proposed a packet scheduling mechanism in which the video packets are scheduled for transmission through multiple paths according to the importance of the video frames in each group of picture (GOP). Highest priority is given to the packets containing frames with type I-frames (intra coded picture) and P-frames (predictive coded picture), then B-frames (bidirectional predictive coded picture). It has proposed a multipath video delivery based on packet and path priority that collects information about the paths by transmitting control packets over the WMSN periodically. It classifies packets according to their types and more important packets are transmitted through paths with better conditions. For this purpose, three queues are used for each kind of video frame packets and different priorities are given to each queue at the source.

The authors of [10] have proposed a mechanism for calculating paths score according to paths properties and QoS requirements of the multimedia applications. The proposed scheme in [11] is based on sending video packets over two disjoint paths besides using buffering technique in 
special nodes of the network. In each path one node is selected as cache node. The main task of the cache nodes is to realizing different packet types, buffering some of important video packets, reducing forward traffic rate while detecting loss in network and local error management to overcome high loss rate of the video packets.

In [9], a power efficient multipath video transmission scheme has been proposed that selects less important video packets to be dropped by using a recursive distortion prediction model. It provides a power aware packet scheduling that is able to identify the available paths from the source to the receiver and schedules packets for transmitting over the selected paths according to energy efficiency of the participating sensor nodes in the paths. The authors of [12] have proposed a multipath routing protocol which can discover multiple paths with high energy efficiency. The work in [13] has proposed using different metrics for multipath computation for different video encoding schemes (e.g. Multiple Description Coding and Layered Coding). Furthermore, it has adopted a more practical measurement technique for predicting the quality of a link for improving path selection for video streaming.

In [14], video stream data is partitioned into image and audio streams and two kinds of priorities are given to them for using of limited bandwidth and energy in the network based on application requirements. The authors of [15] have used path priority scheduling and cross-layer technique for adaptive video coding to path status with frame skipping, reference frame selecting and intraframe refreshing techniques for H.26L real-time video streaming over WSNs.

Single path video data transmission in wireless sensor networks causes the energy of the sensor nodes throughout the path to be consumed and consequently the network be partitioned. In addition, transmission of video data through a single path leads to congestion in the intermediate nodes that causes increasing the packet loss rate and end-to-end delay. It seems using multipath transmission for video data is more efficient than single path approach [5].

Some main advantages of using multipath routing techniques for video transmission over sensor networks are uniformly load distribution, improving the reliability, expanding the bandwidth, and delay reduction. Since our packet scheduling scheme in this paper is based on multipath routing in wireless sensor networks, we briefly discuss AOMDV as a sample of multipath routing protocols.

AOMDV is the multipath version of the AODV routing algorithm [19]. This protocol intends to detect both link-disjoint and node-disjoint paths that conduce to the destination. In order to support the multipath routing in AOMDV, all routing tables contain a list of paths for each destination. All paths that conduce to one destination have same sequence number. For loop prevention in the network and also creation of disjoint paths, in this protocol two values should be saved for each path, the distance from the source node and the last hop. The value of data filed for the distance from the source node will not be changed until a path for the next sequence number is specified.

To find node-disjoint routes, each node does not immediately reject duplicate RREQs (Route Request). Each RREQs arriving via a different neighbour of the source defines a node-disjoint path. This is because nodes cannot broadcast duplicate RREQs, so any two RREQs arriving at an intermediate node via a different neighbour of the source could not have traversed the same node. In an attempt to get multiple link-disjoint routes, the destination replies to duplicate RREQs, the destination only replies to RREQs arriving via unique neighbours. After the first hop, the RREPs follow the reverse paths, which are node disjoint and thus link-disjoint $[18,19]$. The advantage of using AOMDV is that it allows intermediate nodes to reply to RREQs, while still selecting disjoint paths. AOMDV has more message overheads during route discovery due to increased flooding and multiple RREQs replies by the destination [18]. 


\section{The Proposed Priority Scheduling Method}

When there is huge amount of traffic in the network, using multipath routing cannot provide the required quality for the perceived video alone. Thus, an additional mechanism such as scheduling will be needed. The role of such an additional mechanism is to give appropriate priority to each video frame in conjunction to the other data in order to avoid frame losses while achieving QoS for video streaming.

As we know different video frames in a compressed video stream have different effects on the perceived video quality at the receivers. Reference frames have much more effect on the video quality. Moreover, unsuccessful reception of a reference frame would eventuate the unsuccessful decoding of all dependent frames. Consequently, this causes resource inefficiency in the network. In this section we propose a packet scheduling mechanism for transmission of video packets through multiple paths in the network based on the type of the vide packets. Our proposed solution has three parts: A) Packet scheduling, B) Queue scheduling, C) Path scheduling. In the remaining of this section we explain each part.

\subsection{Packet Scheduling}

Since different video frames in a GOP have different impact of the perceived video quality, it is better to give per frame different priority. In this way, reference frames would have the most priority compared with the other video frames. In our proposed packet priority scheduling, the highest priority is given to the packets that containing frames with type of I-frame (Intra coded picture) and P-frame (Predictive coded picture) then B-frame (Bidirectional predictive coded picture), respectively. Furthermore, the packets containing I-frames must be sent through the most reliable paths in the network.

\subsection{Queue Scheduling}

We also propose a scheduling mechanism for the intermediate nodes in each path. Such a mechanism can improve the video delivery over WMSN. In our queue priority scheduling, nodes' buffer is divided into four queues and a round robin scheduling is executed for them. Each arriving packet to a node is placed on one of the queues according to following rules:

- A packet that contains I-frame is placed in the first queue.

- A packet that contains P-frame is placed in the second queue.

- A packet that contains B-frame is placed in the third queue.

- A packet with no video data is placed in the fourth queue.

The algorithm for enqueuing the packets in the nodes' buffer is shown in Algorithm 1.

The proposed scheduling scheme operates in this way. The packets of three queues that have higher priorities, because of containing video packets, are transmitted with round-robin scheduling. If these three queues are empty and don't have any packets, then the fourth queue is served one packet. It is worth to mention that First-In-First-Out (FIFO) discipline is used for each queue. The proposed queue scheduling mechanism for each node is provided in Algorithm 2. 


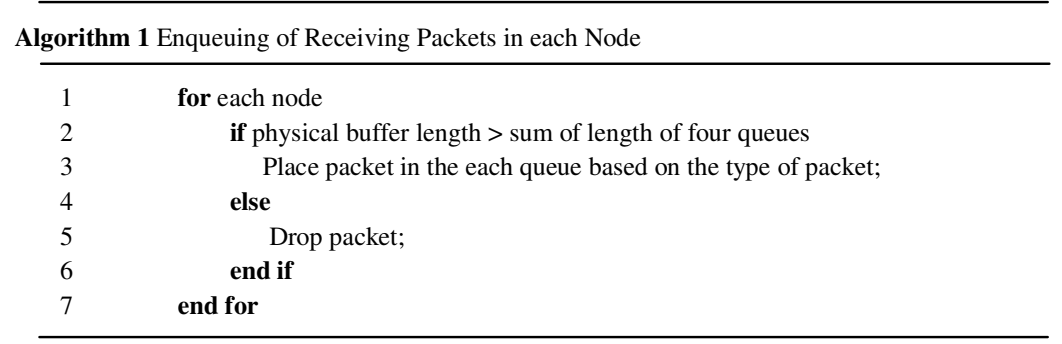

Since in each GOP we have only one I frame and a number of P and B frames, and the number of $\mathrm{B}$ frames is more than that of $\mathrm{P}$ frames, using above queue priority scheduling provides more resources for I frames then for P and B frames, respectively. The other data packets in any node are served if and only if there is not any packet in the video buffers. In this way, the video packets are quickly transmitted compared to other data packets.

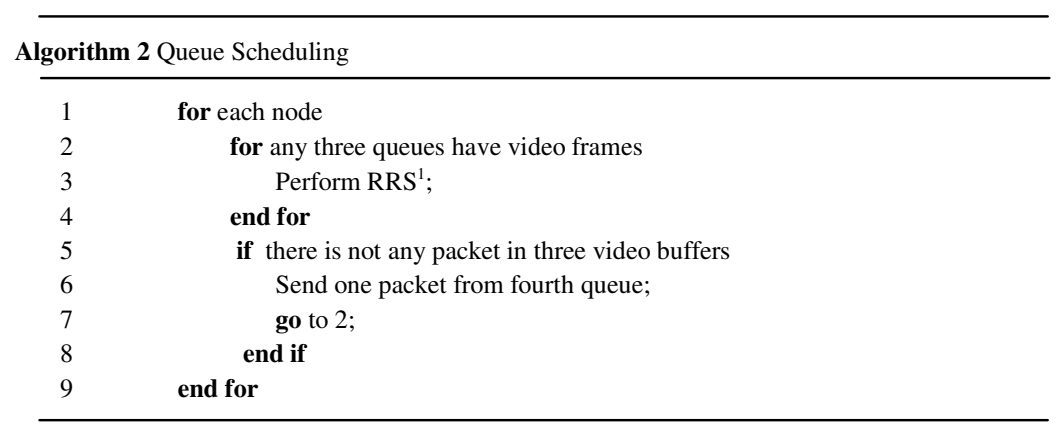

${ }^{1}$ Round-Robin Scheduling

\subsection{Path Scheduling}

Since recognizing high reliability paths for transmission of more important video frames is necessary in our proposed approach, we use control packets for collecting information of each path. Then the path score is calculated based on such information. The multipath routing protocol that we have used is AOMDV [19]. AOMDV creates paths with node-disjoint from video source node to the receiver. In this way, control packets will be sent from all video source nodes to the receiver over all paths in multipath routing. In [10], the authors have proposed a mechanism for achieving the most reliable path with metrics such as delay, bandwidth and energy. The path with the highest score has the best condition for transmitting high priority packets. Each path score is calculated as following $[9,10]$ :

where

$$
\text { Path score }=\alpha W_{L}+\beta W_{E}+\gamma W_{Q}+\lambda W_{H}
$$

$$
\begin{gathered}
\alpha+\beta+\gamma+\lambda=1 \quad 0 \leq \text { Path score } \leq 1 \\
W_{E}=\frac{\text { Min residual energy in the path }}{E_{0}} \\
W_{Q}=\frac{\text { Min Free Buffer size in the path }}{B_{0}} \\
W_{H}=\frac{(1+\text { Max Hop Count-Hop Count })}{\text { Max Hop Count }} \\
W_{L}=1-\frac{\text { number of packets that do not satisfy required delay }}{\text { number of received packet from each path }}
\end{gathered}
$$

Calculating of path score is based on $\alpha, \beta, \gamma$ and $\lambda$ coefficients. Each coefficient affects on its corresponding component importance. Choosing these coefficients is dependent to the considered 
application. In this work, we adopted the values for these coefficients from [10]. Hop Count is the number of hops to destination. Whenever AOMDV, as multipath routing protocol, is executed; Hop Count is calculated. Max Hop Count is the network diameter. Buffer Size is the total number of packets that can be placed in the node buffer initially and Free Buffer Size is the number of packets that can be placed in the node buffer in each time. E0 and B0 are initial energy and buffer size of the intermediate nodes, i.e. the nodes between source and destination in the path. Every control packet has three parameters:

1- Path ID that shows path number

2- Minimum residual energy that shows minimum residual energy of the nodes along the path

3- Minimum free buffer size that shows the minimum free buffer size of the nodes along the path.

Moreover, each intermediate node holds three parameters: hop count, its residual energy and free buffer size. The control packets are sent through the paths, periodically. Each intermediate node that receives a control packet updates its fields and forwards it to the next node. The algorithm for updating the control packets is provided in Algorithm 3.

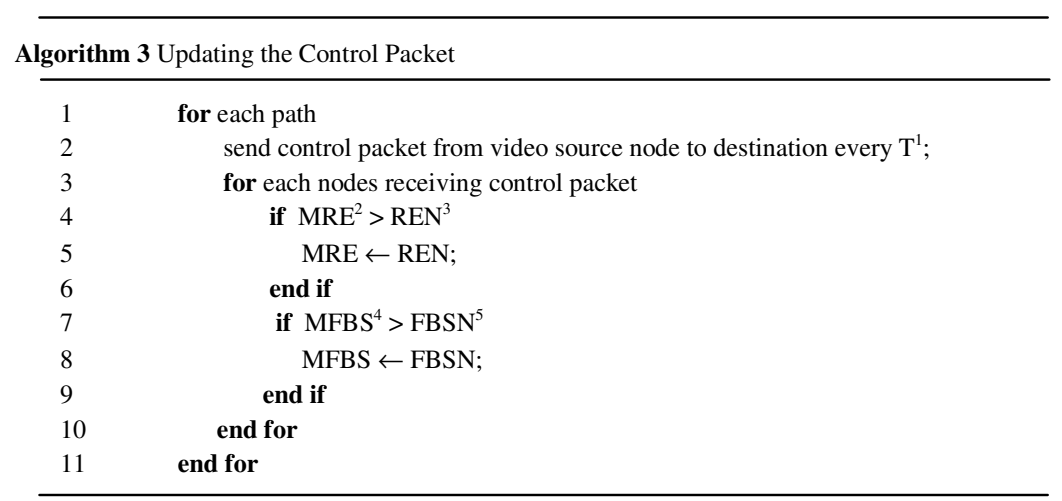

${ }^{1} \mathrm{~T}=20 \mathrm{~s} ;{ }^{2}$ Minimum Residual Energy; ${ }^{3}$ Residual Energy of Node; ${ }^{4}$ Minimum Free Buffer Size; ${ }^{5}$ Free Buffer Size of Node

Video packets that don't satisfy delay requirement and aren't received in their deadline will be considered as lost packets. End-to-end delay in each path is calculated as following [14]:

$$
D_{e 2 e}=D_{H O P} \cdot H
$$

where, $D_{H O P}$ is average delay of each hop and $H$ is the number of hops in each path. Since network conditions vary over time, control packets are transmitted from source to destination periodically for collecting paths information and calculation of paths scores. Upon receiving a control packet by the receiver, path score is calculated based on hop count, minimum residual energy, minimum free buffer size and video packet loss based on delay parameter for each path. Then path score is sent from destination to video source node by using feedback channel. In video source node, paths are sorted according to their scores and the path with the higher score is considered as a reliable one for transmitting more important video frames. Thus video packets with higher priority will be sent to paths with higher scores. This causes more important packets to be transmitted through more reliable paths and therefore it could increase video quality at the receiver. The algorithm for calculating path score and sorting path is listed in Algorithm 4. 


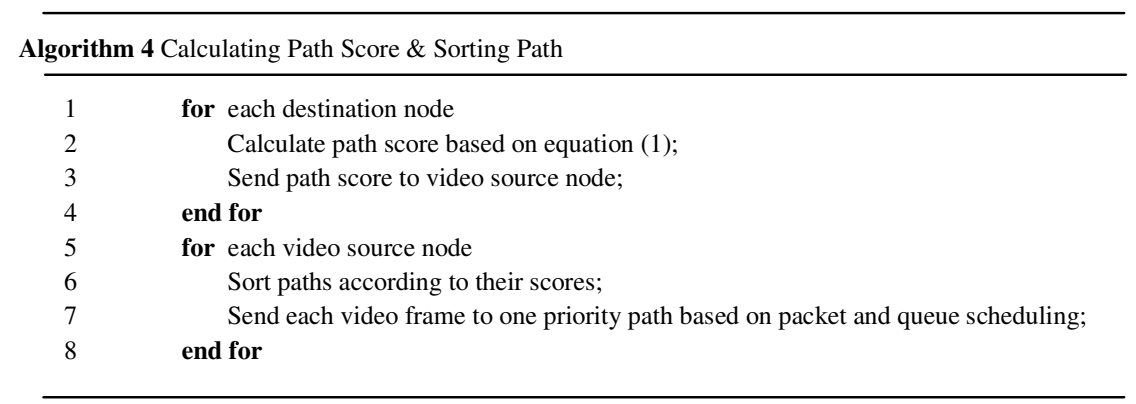

In this scheduling method, if there is not any most important video packet (e.g. I-frame packets) for transmitting in the source node, then video packets with lower priority will be transmitted towards the path with highest quality. In this way, we use packet and queue scheduling mechanism for video transmission in the source node. Diagram of the proposed video transmission method is shown in fig. 1.

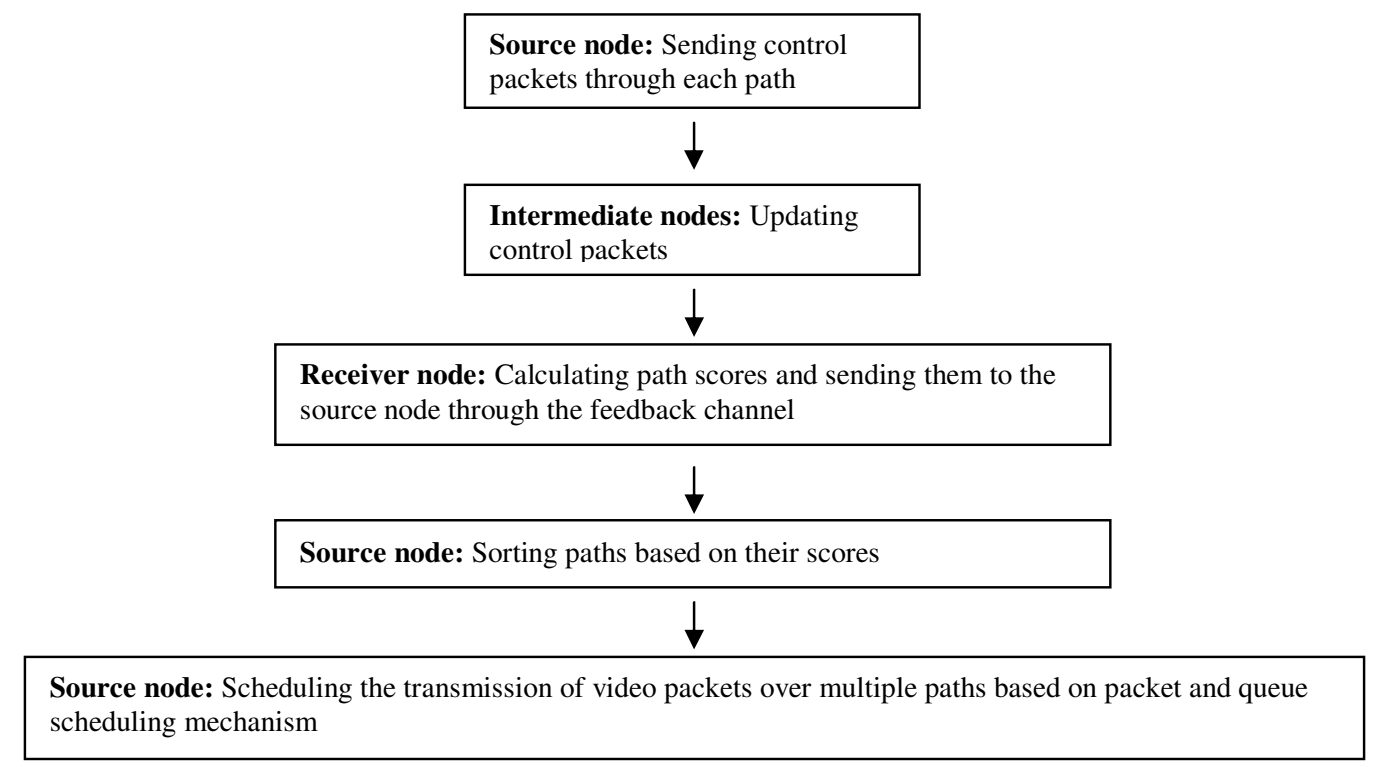

Figure 1. Diagram of the proposed video transmission

\section{Simulation ReSUlts}

\subsection{Simulation Setup}

For performance evaluation of our proposed method through simulation we used NS2 network simulator [20]. Moreover, we generated MPEG4 [21] video traffic by using EvalVid [22, 23, 24] frame work. In this frame work we used ffmpeg [25] encoder. Our used performance metrics are end-to-end delay, cumulative jitter, frame loss rate and etc. Our used simulation setup is shown in Table I.

In our simulation results we incorporate queue priority scheduling with packet and path priority scheduling over AOMDV routing protocol. The first control packets are transmitted 50 seconds 
after execution of AOMDV and control packets request interval was $\mathrm{T}(\mathrm{T}=20 \mathrm{~s})$. For this purpose, we transmitted Paris video sequence, with CIF (i.e. 176*144) format and 1065 frames, and analyzed the results.

TABLE I. PARAMETERS OF THE SIMULATION SETUP

\begin{tabular}{|l|l|}
\hline Parameters & Value \\
\hline Network Dimension & $1000 \mathrm{~m} \times 1000 \mathrm{~m}$ \\
\hline Number of Nodes & 150 \\
\hline Sensor Initial Energy & 1000 Joules \\
\hline Node Placement & Uniformly Distributed \\
\hline Traffic & $\begin{array}{l}\text { Two nodes send video traffic to the base station } \\
\text { and each other node sends } 400 \text { bps traffic to the } \\
\text { base station. }\end{array}$ \\
\hline Buffer Size & 200 Packets \\
\hline Packet size & 400 Bytes \\
\hline Average Hop Delay & $20 \mathrm{~ms}$ \\
\hline $\begin{array}{l}\text { Control Packet } \\
\text { Request Interval }\end{array}$ & $\mathrm{T}=20 \mathrm{~s}$ \\
\hline
\end{tabular}

\subsection{Experiment Results}

\subsubsection{PSNR}

Fig. 2 shows the overall PSNR of video frames in AOMDV with random scheduling for video streaming compared to AOMDV with proposed scheduling at the source and the intermediate nodes. Using packet and path priority scheduling provides high improvement in PSNR of the received video. Also queue priority scheduling giving high priority for video packets against other packets. In addition, using weighted fair queuing in the intermediate nodes causes the packets belonging to more important video frames (i.e. I and P frames) to be forwarded more quickly than other ones and consequently decreasing the end-to-end delay and video packet loss at the receiver. Decreasing end-to-end delay and packet loss of video traffic obviously improves the PSNR of the received video.

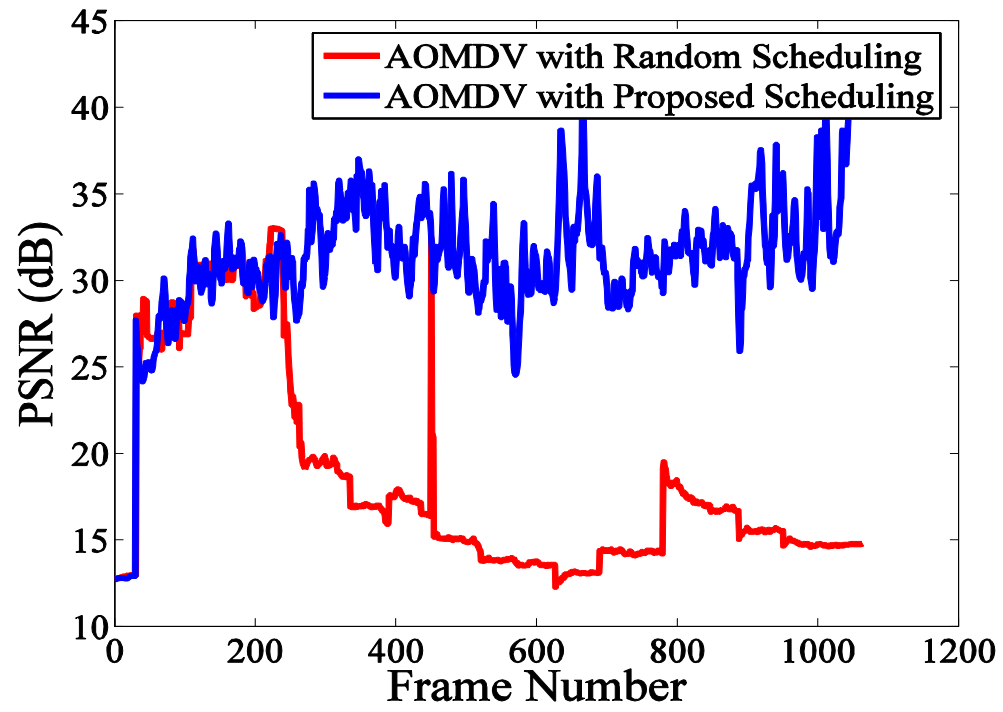

Figure 2. PSNR of video frames for AOMDV and the proposed scheduling 


\subsubsection{Frame Loss}

Fig. 3 compares percentage of frame loss in random scheduling with proposed scheduling. Decreasing lost video frames in our scheduling can causes a high improvement in QoS in realtime video. The reason is that the proposed scheduling scheme tries to reduce the loss of the video packets in the networks by recognizing the most reliable paths for transmitting the reference video frames that have the most effect on the received video distortion.

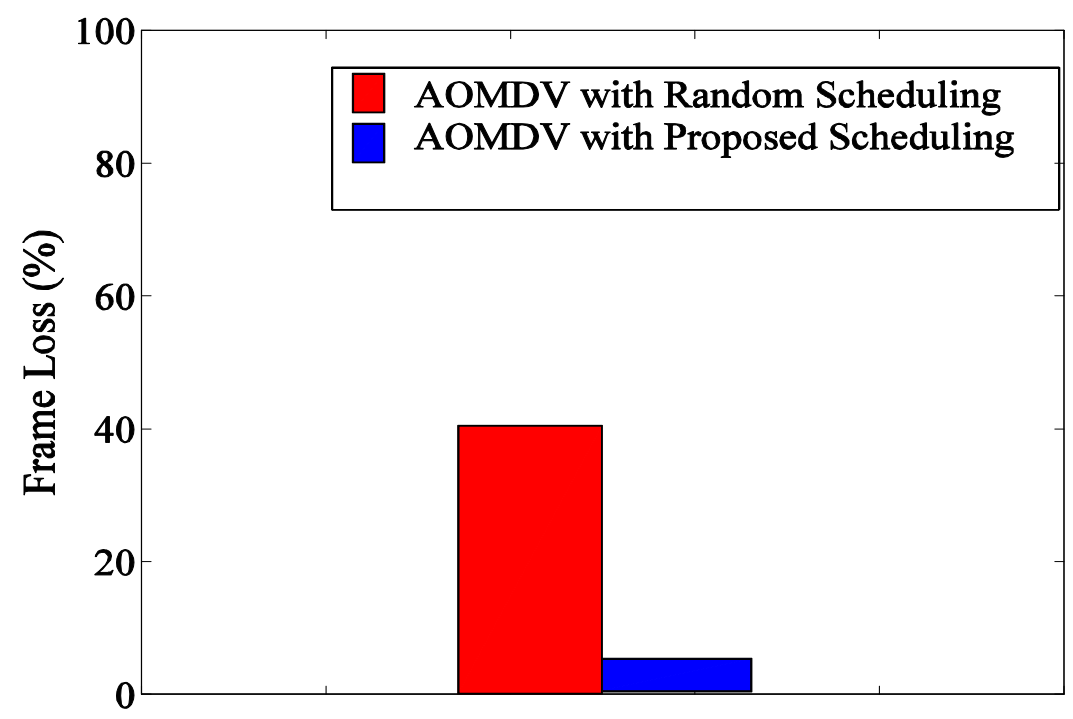

Figure 3. Video frame loss in AOMDV and the proposed scheduling

Play-out buffering is used at the receiver for jitter removal of the video traffic in the network. Big play-out buffering time can reduce the video frame loss resulted from late arrivals of the video packets while increasing the start-up play time. In order to study the impact of the pay-out buffering time on frame loss rate at the receiver, We examined different values of Play-out buffering time such as $1200 \mathrm{~ms}, 1400 \mathrm{~ms}$ and so on. For greater play-out buffer times, video frames have more time to be received at the receiver. Fig. 4 shows the percentage of frame loss against play out buffering time in this experiment. As shown in the figure by increasing the playout buffering time, the frame loss rate is decreased in both methods, but the decreasing rate in our proposed method is considerably more than the random scheduling. This is due to giving high priority to more important video packets and also sending the reference frames through more reliable paths that consequently lead to decreasing in the late arrival loss at the recover. Fig. 4 shows that in high play out buffer time, such as $1500 \mathrm{~ms}$ and after that, the value of frame loss rate for our priority scheduling is as low as $4.79 \%$ whiles the value of frame loss rate with random scheduling is about of $55 \%$ time. 


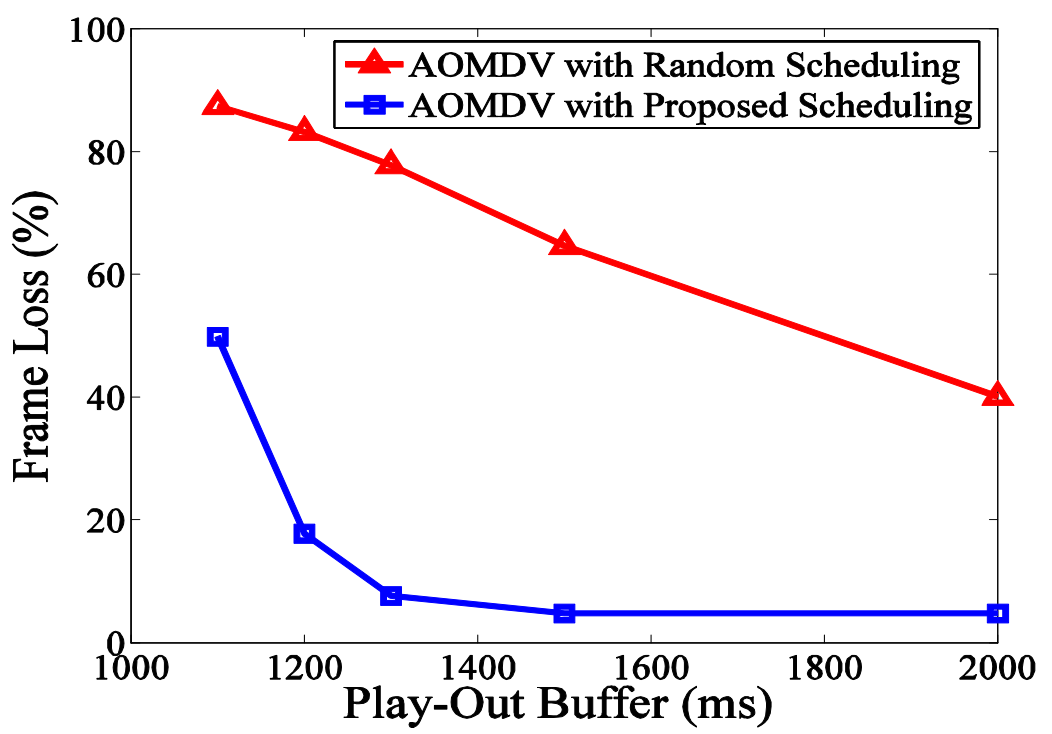

Figure 4. Video frame loss in AOMDV and the proposed scheduling against play-out buffering time

\subsubsection{End-to-End Delay}

End-to-end delay and cumulative jitter for per frame at the receiver are generated with EvalVid $[22,23]$. In video transmission systems not only end-to-end delay is important for the perceived video quality, but also the variation of the delay, usually referred to as frame jitter, is important for the video quality. Fig. 5 shows end-to-end delay in AOMDV with random scheduling and AOMDV with the proposed scheduling. Using packet and path priority scheduling in the video source node, the reliable paths are dedicated to important video packets in networks. Also, the proposed queue scheduling can achieve a high improvement in end-to-end delay. The proposed mechanism transmits video packets just and quickly in proportion of the other packets. This is equivalent to giving priority to video frames when there is a huge amount of CBR data traffic in network. Thus, in this way with decreasing queuing delay for video packets in the buffer of intermediate nodes, the end-to-end delay of frames will be decreased. 


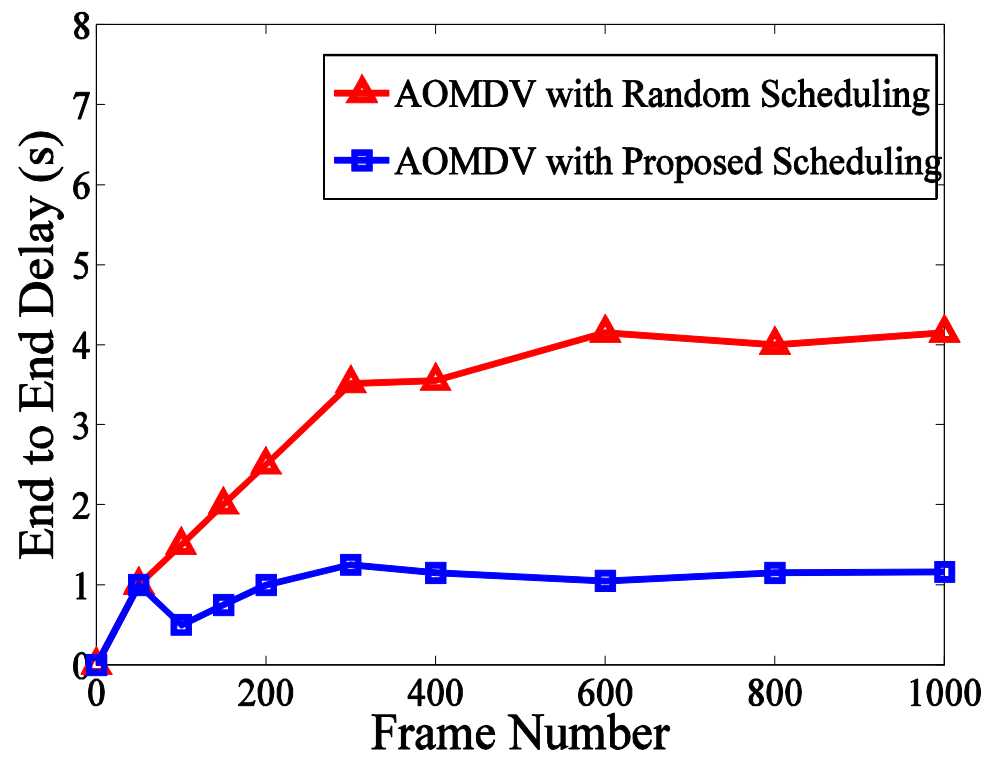

Figure 5. End-to-end delay for video frames in AOMDV and the proposed scheduling

Fig. 6 shows cumulative jitter in AOMDV with random scheduling and AOMDV with the proposed scheduling. Our scheduling can produce a high improvement in cumulative jitter. Our mechanism, through using scheduling in intermediate nodes, fairly and quickly transmits video packets compared with data packets. Also, using packet and path priority scheduling in the video source node, the reliable paths are dedicated to important video packets in networks. Thus, in this way, through decreasing end-to-end delay and reliable transmitting for video packets, cumulative jitter of frames improves.

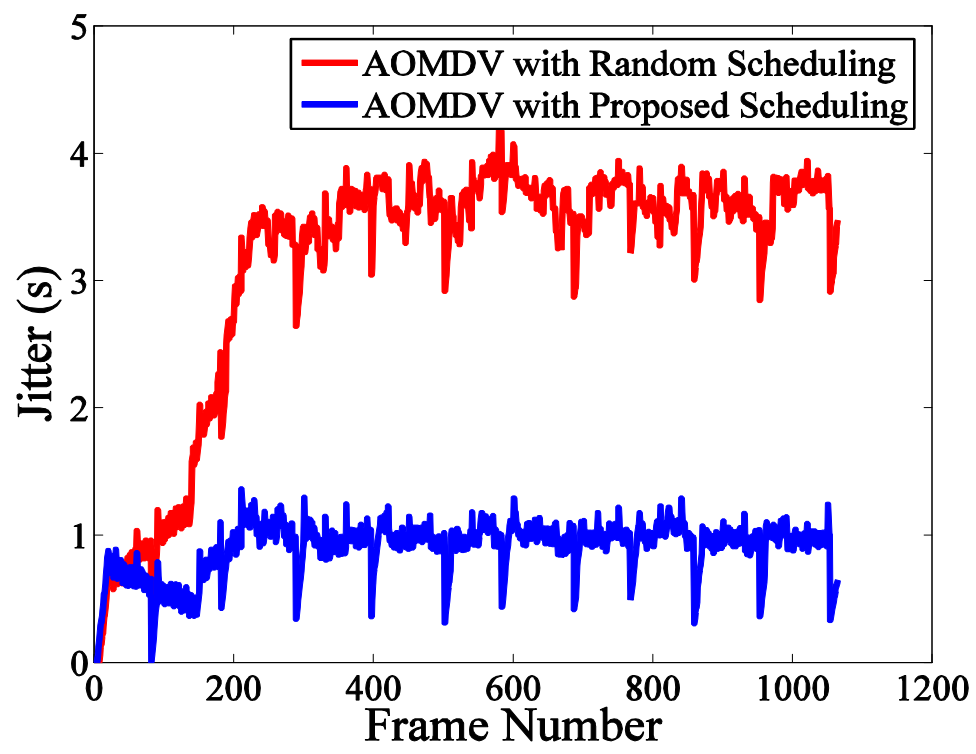

Figure 6. Cumulative jitter for per frame in AOMDV and the proposed scheduling 


\subsubsection{Inter Frame Gap}

High inter frame gaps in the received video cause freezing, and consequently decrease the video quality. Fig. 7 shows inter frame gap in AOMDV with random scheduling and AOMDV with the proposed scheduling. This results show that the proposed scheduling mechanism can achieve high improvement in inter fame gap of the received video at the receiver.

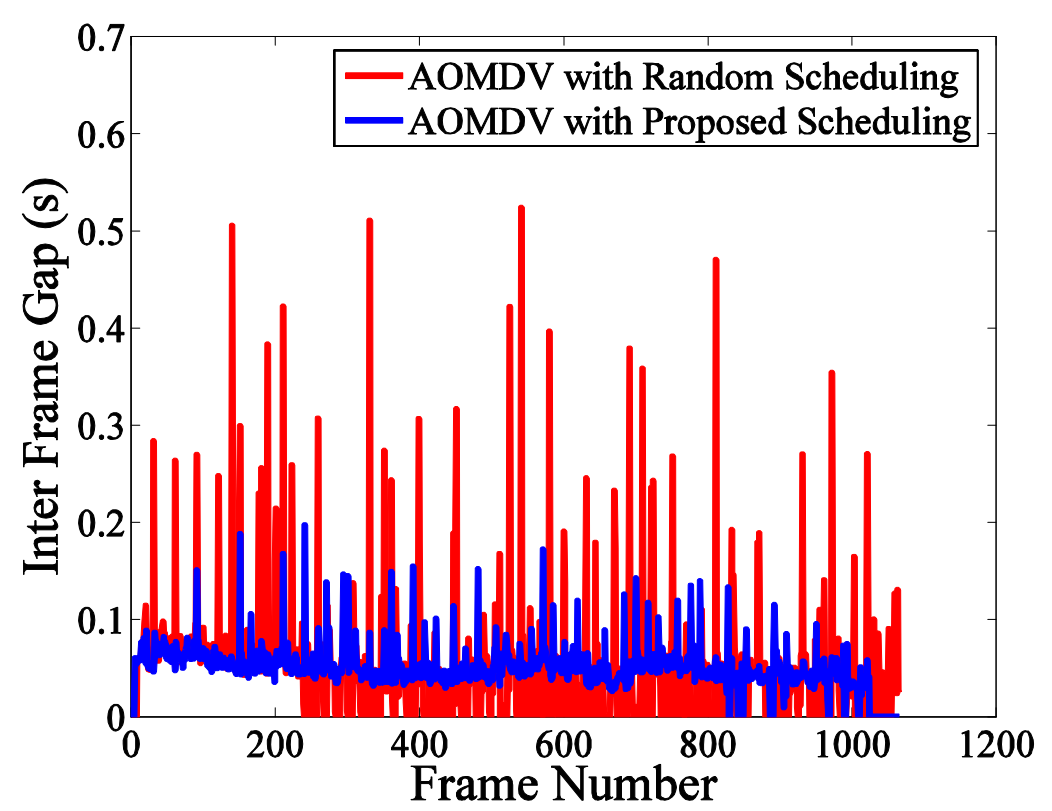

Figure 7. Inter frame gap for AOMDV and the proposed scheduling

Fig. 8 presents video quality at the receiver for using AODV with random scheduling, AOMDV with random scheduling and AOMDV with the proposed scheduling. As the results shows, using single path routing cannot be efficient for video streaming in WMSNs, while in multipath routing, correlation among packet losses, with transmitting video frames over several paths, is reduced. In addition, using efficient scheduling mechanism along with multipath routing can achieve better results.

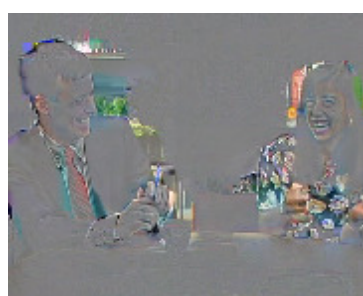

(a)

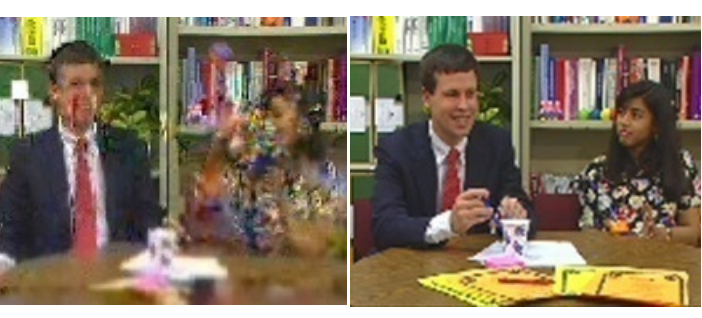

(b) (c)

Figure 8. Paris sequence (a) using AODV with random scheduling (b) using AOMDV with random scheduling (c) using AOMDV with the proposed scheduling

\section{Conclusions}

Video delivery with high quality over a WMSN that have limited resources, such as energy, is a challenging problem. In this paper, we proposed a new mechanism for improving video delivery over WMSN in which all of the nodes participate in priority scheduling. In the video source node, 
packet, queue and path priority scheduling are used. Using queue priority scheduling, different types of video frames will be treated according to their importance. With packet and path priority scheduling, high priority video packets are transmitted over high reliable paths. For this goal we used control packets that are transmitted from the video source node over the routing paths for collecting information of each path. Since network condition may vary over the time, control packets are periodically transmitted from source to destination for collecting paths information. The simulation results show that our proposed scheduling solution improves the quality of the perceived video at the receiver, considerably.

\section{REFERENCES}

[1] I.F. Akyildiz, T. Melodia, K.R. Chowdhury, (2007) "Wireless multimedia sensor networks: A survey," IEEE Wireless Communications 14, no. 6, pp. 32-39.

[2] I.F. Akyildiz, T. Melodia, K.R. Chowdhury, (2007) "A survey on wireless multimedia sensor networks," Computer Networks 51, no. 4, pp. 921-960.

[3] M.H. Hajiesmaili, A. Khonsari, M.S. Talebi, (2012) "Content-aware rate allocation for efficient video streaming via dynamic network utility maximization," Journal of Network and Computer Applications Volume 35, Issue 6, pp. 2016-2027.

[4] J. Guo, L. Sun, R. Wang, (2012) “ A Cross-layer and Multipath based Video Transmission Scheme for Wireless Multimedia Sensor Networks," Journal of Networks, Vol 7, No 91334-1340.

[5] J. Nightingale, Q. Wang, C. Grecos, (2013) "Performance Evaluation of Concurrent Multipath Video Streaming in Multihomed Mobile Networks," International Journal of Digital Multimedia Broadcasting Volume 2013 Article ID 319594, 20 pages.

[6] I. Politis, M. Tsagkaropoulos, T. Dagiuklas, S. Kotsopoulos, (2008) "Power Efficient Video Multipath Transmission over Wireless Multimedia Sensor Networks,” Mob. Netw. Appl. 13, no. 3-4, pp. $274-$ 284.

[7] JN Al-Karaki, AE Kamal , (2004) "Routing techniques in wireless sensor networks: a survey," Wireless Communications, IEEE, vol. 11, no. 6, pp. 6-28.

[8] K. Akkaya, M.Younis; (2003) "An Energy-Aware QoS Routing Protocol for Wireless Sensor Networks," IEEE, pp. 710- 715.

[9] A.R. Lari, B. Akbari, (2010) "Network-Adaptive Multipath Video Delivery over Wireless Multimedia Sensor Networks Based on Packet and Path Priority Scheduling," IEEE International Conference on Broadband, Wireless Computing, Communication and Applications.

[10] H. Yousefi, A. Dabirmoghaddam, K. Mizanian, A.H. Jahangir, (2009) "Score Based Reliable Routing in Wireless Sensor Networks," IEEE 23rd International Conference on Information Networking.

[11] P. Panahi, (2010) "The Feedback Based Mechanism for Video Streaming Over Multipath Ad Hoc Networks," Journal of Sciences, Islamic Republic of Iran 21(2), pp. 169-179.

[12] Ke Guan, Li-Ming He, (2010) “A Novel Energy-Efficient Multi-Path Routing Protocol for Wireless Sensor Networks," IEEE International Conference on Communications and Mobile Computing.

[13] S. Murthy, P. Hegde, V. Parameswaran, B. Li, and A. Sen, (2007) "Improved Path Selection Algorithms for Multipath Video Streaming in Wireless Ad-Hoc Networks," IEEE International Conference,

[14] L. Zhang, M. Hauswirth, Z. Zhou, V. Reynolds, and G. Han, (2008) "Multi-priority Multi-Path Selection for Video Streaming in Wireless Multimedia Sensor Networks," In the fifth International conference on Ubiquitous Intelligence and Computing (UIC 2008), Oslo, Norway, pp. 23-25.

[15] Min Chen, Victor C.M. Leung, Shiwen Mao, Ming Li, (2008) "Cross-layer and Path Priority Scheduling based Real-time Video Communications over Wireless Sensor Networks," IEEE vehicular technology conference, Singapore, VTC spring, pp. 11-14.

[16] P. Hurni,T. Braun, (2008) "Energy-Efficient Multipath Routing in Wireless sensor network, " Springer, vol. 5198, pp. 72-85.

[17] C. E. Perkins, E. m. Royer, and S. R. Dos, (2000) "Ad hoc on Demand Distance Vector (AODV) Routing," IETF Internet Draft.

[18] S. R. Biradar, K. Majumder, S. K. Sarkar, Puttamadappa C, (2010) "Performance Evaluation and Comparison of AODV and AOMDV, " International Journal on Computer Science and Engineering (IJCSE), vol. 02, no. 02, pp. 373-377. 
International Journal of Computer Networks \& Communications (IJCNC) Vol.5, No.6, November 2013

[19] Mahesh K. Marina, Samir R. Das, (2001) "On-demand multipath distance vector routing in ad hoc networks," Ninth International Conference for Network Protocols (ICNP).

[20] Information Science Institute, NS-2 network simulator, Software Package, 2003, http://www.isi.edu/nsnam/ns/

[21] MPEG4 Encoder, http://megarea.ee.nctu.edu.tw/mpeg.

[22] J. Klaue, C. K. Shieh, W. S. Hwang, and A Ziviani, (2008) "An Evaluation Framework for More Realistic Simulations of MPEG Video Transmission," Journal of Information Science and engineering, vol. 24, no. 2, pp. 425-440.

[23] J. Klaue, B. Rathke, and A. Wolisz, (2003) "EvalVid - A Framework for Video Transmission and Quality Evaluation," in Proc. of the 13th International Conference on Modelling Techniques and Tools for Computer Performance Evaluation, Urbana, Illinois, USA.

[24] Integrating EvalVid with NS2, http://140.116.72.80/ smallko.

[25] ffmpeg's Official Webpage, http://ffmpeg.mplayerhq.hu.

[26] AOMDV in NS2, http://wpage.unina.it/marcello.caleffi/ns2/aomdv.

\section{Authors}

Elham Karimi received BE and MS degrees from the Department of Electrical and Computer Engineering, Qazvin Islamic Azad University, Qazvin, Iran in 2007 and 2011. Her research interests are in video streaming, wireless networking, and computer architecture. She is working as university instructor.

Behzad Akbari received BE, MS and degrees from the Department of Computer Eng., Sharif University of Technology, Tehran, Iran in 1999, 2002 and 2008. He is assistant Professor at Tarbiat Modares University, Tehran,Iran. http://www.modares.ac.ir/en/Schools/ece/academic-staff/ b.akbari/1
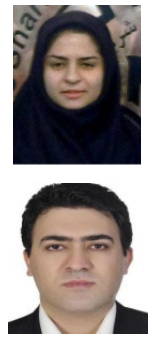